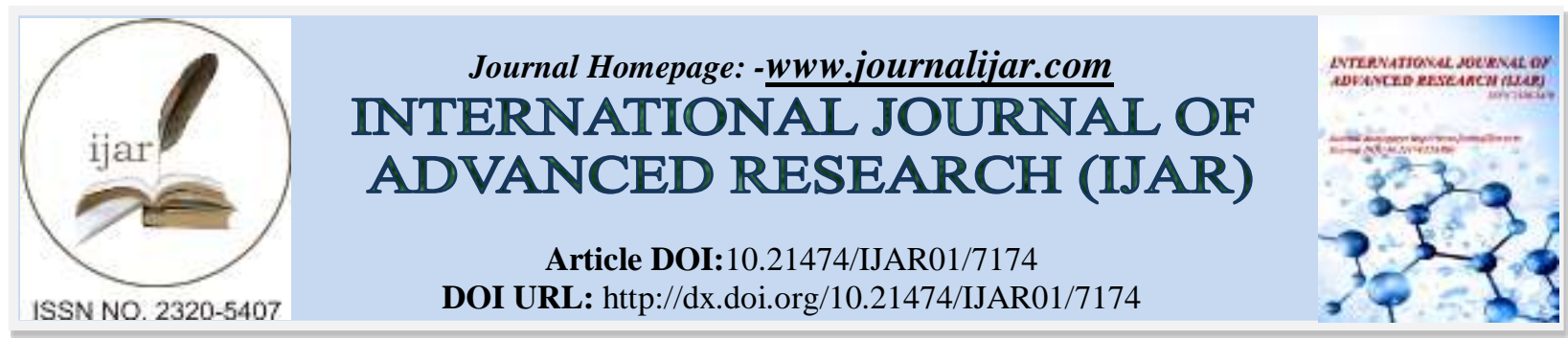

RESEARCH ARTICLE

\title{
A NOVEL SCHEME FOR CONTINUOUS MONITORING OF KIDNEY PATIENTS.
}

\author{
Rahul Chauhan $^{1}$, Sumeet Chandel ${ }^{1}$, Varun Sharma ${ }^{1}$, Aanchal Khatri ${ }^{2}$ and Jayati Ralen ${ }^{1}$. \\ 1. Manav Rachna University, Faridabad, India. \\ 2. Accendre KMS Ltd.
}

\section{Manuscript Info}

Manuscript History

Received: 22 March 2018

Final Accepted: 24 April 2018

Published: May 2018

Keywords:-

Kidney Patients, Internet of things,

Wearable Device.

\begin{abstract}
Internet of things is been a major field of research in smart health care system due to its vast advantages like keeping a detailed record of patient health, remote sharing of data and its ability to connect with cloud to provide almost never ending storage. The combination of using cloud with internet of things is referred as cloud of things (COT). This paper talks about the technologies ubiquitous for smart healthcare system and there advantages, accuracy and challenges. This paper focus on cloud computing, machine learning, wearable sensors, radio frequency identification (RFID) and security for remote sharing of patients data. This paper presents a future model for IOT healthcare system.

Copy Right, IJAR, 2018,. All rights reserved.
\end{abstract}

\section{Introduction:-}

Internet of things is a system of physical devices, person, animal or any object that has embedded sensors, software etc to communicate each other over a network. this is recognized as one of the most important area of future technology with more advancement in technology this will be an integration of cloud computing and IOT. Cloud has the availability of unlimited storage and processing power. This paper talks about the field of medical science and smart hospital system.

In developing countries there are area where people don't have adequate access to proper healthcare system. A recent study conducted in countries like Australia, Canada, Germany, New Zealand, the United Kingdom and the United States shows that patients didn't have proper health records which caused problems in identification of symptoms and diagnosis of disease. Further after discharge no system for follow up with doctor was arranged.

Health is an important aspect of life and with every sunrise a new disease is introduced. With increase in population the demand for doctors and hospital facilities are also increasing. It is also important that we provide quality healthcare system with limited error and ease of patient, doctor and caregiver.

The steadily ageing population and there related rise in chronic illness has put a significant pressure on healthcare system. There is lack of bed in hospitals, insufficient doctors and resources to cater such large number of patients. IOT has emerged as a solution to reduce the pressure on current healthcare system. IOT enables remote sensing of patient by using various hardware and software components. Patients, doctor and caregiver can access patient's data from anywhere and at any time and can be alerted in case of any emergency. Kidney is an important part of human body. It is a bean shaped organ which helps to filter out waste and excess fluids from human body through urine. Loss in kidney function means the waste is build up in body and can further damage other organs of body and can 
even cause heart attack. According to a survey conducted by American kidney fund, kidney disease is the $9^{\text {th }}$ leading cause of death in the united states. About 31 million people in united states suffer from chronic kidney disease. Leading cause of kidney failure are diabetes and blood pressure.

This paper focus on various technologies being used in IOT healthcare It is important that we establish a smart infrastructure for hospital and for proper and reliable functioning of the devices for patients but hospitals too. This paper also discusses some of the area of medical science in which its applications can be of great advantage. Further a model is proposed for detection of kidney failure.

\section{Literature Review:-}

In this paper we are going to highlight some recent strategies used in IOT in smart health care such RFID, cloud computing, big data and many other techniques that can be used by IOT to improve the bonding of doctor-patients so that there will be no misconception . we will also showing some key points of recent research done by people in this field.

(Cristina Elena Turcua, Cornel Octavian Turcua):-[1]

Presented that information and technology hold promise for increasing the accessibility of medical and it important for patient's safety. They surveyed the hospitals of Australia, Canada, New Zealand, the United Kingdom and the United States and found that many patients after getting discharged didn't know the symptoms of the disease that they had and this result in no future communication with the doctor which reduced the bonding of the doctor patient relationship. They found that many recent technologies such as RFID have emerged as a great tool in recognizing the disease or help the patient and doctor to make contact at a interval of month. They also suggested that RFID can be also used to identify patients and what type of disease they have using a tag that will be inserted or used as a wearable device such a wrist watch or any other that can be used with ease. So, this research aims to integrate multiagent and RFID technologies into the IOT platform for health care. So to assist user to how to use this technology some agents will be needed which help in strengthens the bonding of doctor-patient. They also share their objective that It is our objective to demonstrate the strengths and weaknesses of our multi-paradigm approach for the Internet of Things development applied in healthcare.

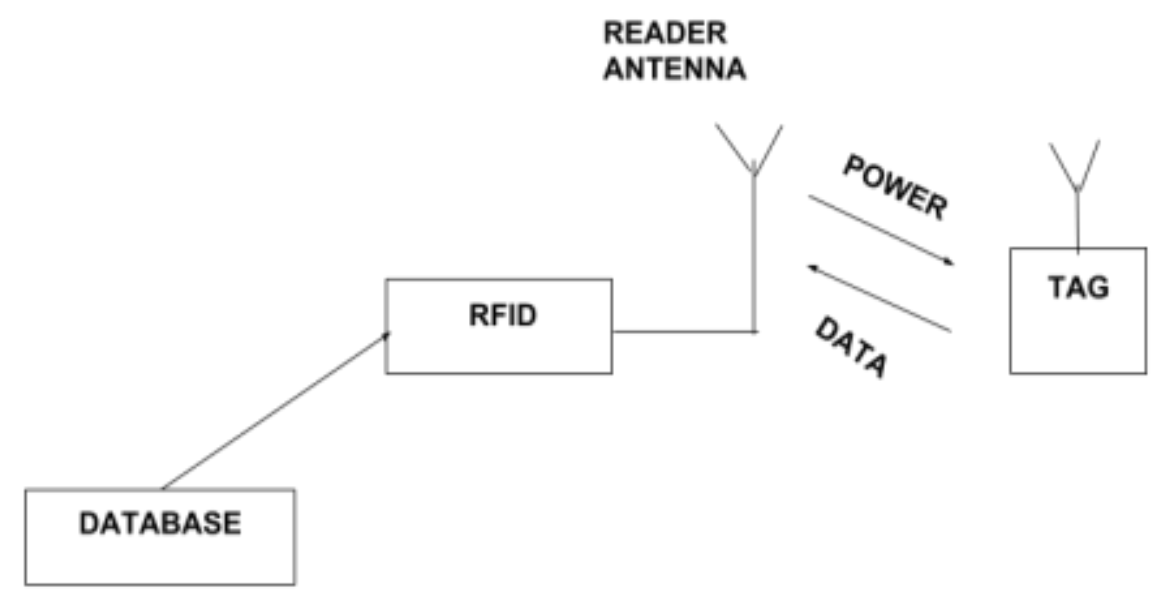

Fig 1:-RFID using tags

\section{(Mohammad Aazam1, Pham Phuoc Hung2, Eui-Nam Huh3):-}

[2] discusses the ubiquitous trend going on computing, everything is going to be connected to internet and its data will be used for various need so, to make the storage of data easy they connected both IOT and cloud computing together and form a new term called Cloud of thing or COT which states that all the data that a consumer uses will not be physically stored but instead of that it will be stored on cloud and making it easy for the user to access though it at any time as this is the beginning of the era of internet everything is going to be connected to internet and soon even the lock of our home will be controlled by the internet and there will be some security issue in this which will be needed to take care of, and a security team will be needed to secure privacy of the user so that no data can be lost or misused by someone. They also stated that some IOT devise will be needed special type of service and storage and application and this will be a potential future work. 


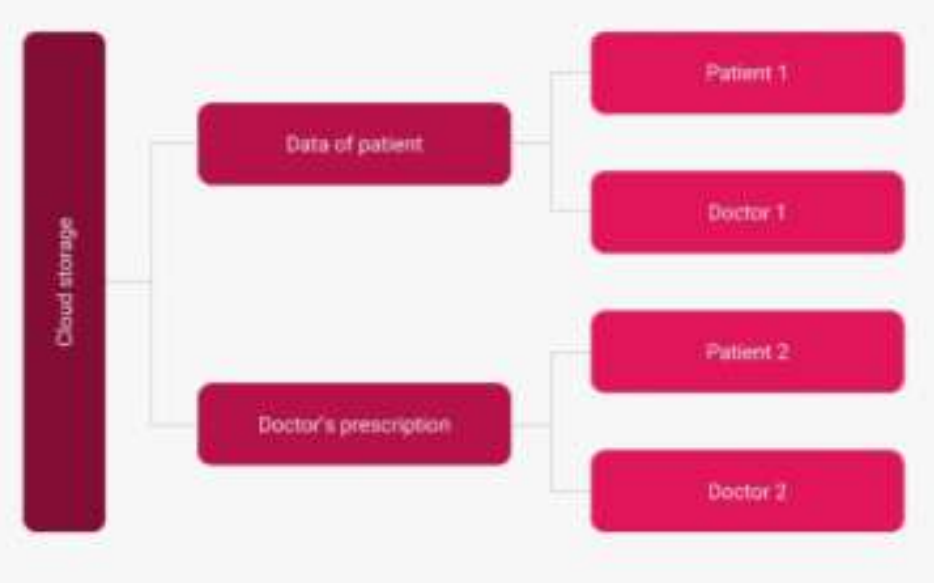

Fig2:-Cloud as a means of storage

\section{(Stephanie B Baker,Wei iang,Ian Atkinson):-}

[3]discuss the importance of IOT in smart healthcare system. Increase in the population has put pressure on the doctors, availability of beds and caregivers in healthcare system. In certain rural areas proper treatment of patients is not available. The author has also discussed about various sensors like pulse rate sensor, respiratory rate sensor, blood temperature sensor etc with their working which are considered as vital signs for human health. The paper discusses various models developed for Parkinson disease, blood glucose level and heart attacks. Many communication standards exist but the most commonly used ones are Bluetooth low energy and ZigBee. This paper gives a great detail about these standards. Machine learning can be very valuable in healthcare system. A great amount of research needs to be done in this field. Machine learning algorithms can help doctors to detect various diseases and plan for better treatment. The paper also addresses cloud as viable solution for storing huge amount of patient data. Author focuses on developing blood pressure monitor which is wearable and has accuracy with respect to hospital standards in their future work.

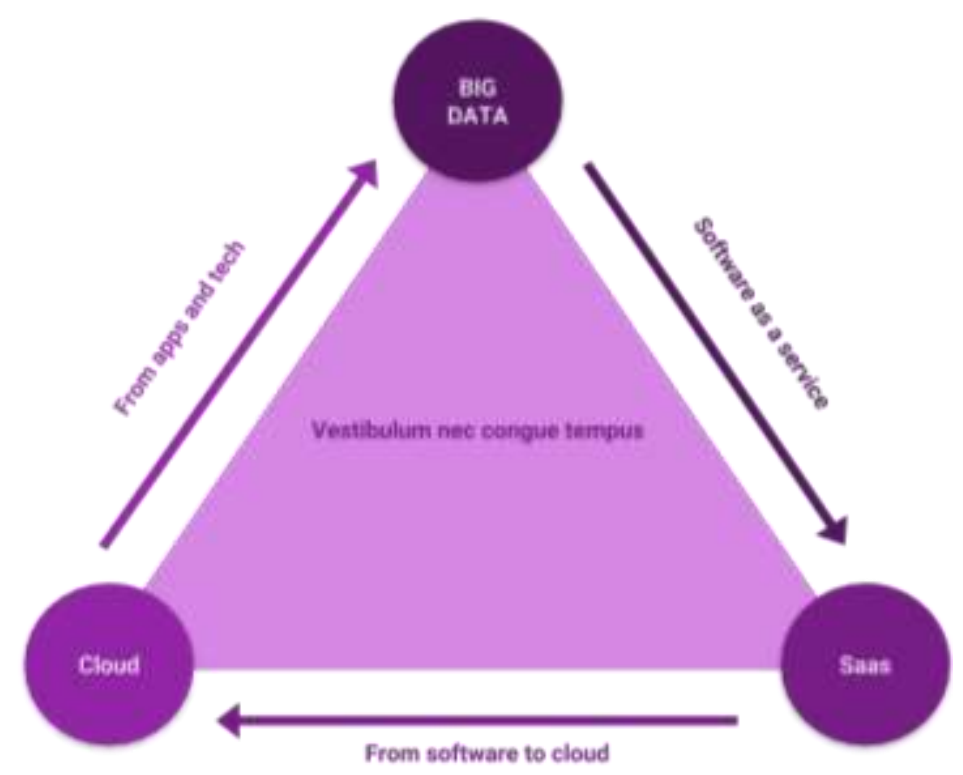

Fig3:-Use of big data in smart health care

(Daniele Miorandi , Sabrina Sicari , Francesco De Pellegrini , Imrich Chlamtac):-

[4] This paper addresses some of the research areas and challenges in IOT. Advancement in technology has led to circuits and battery which can operate in small space environment. These can be easily embedded in smart health care bands and other wearable technologies but their accuracy is the main concern. This paper also discusses the 
importance of Nano technology in IOT for developing small scale components capable of communicating over internet. Each object in IOT should be uniquely identifiable. This can be done by using the RFID tags or QR codes so that they can be easily read. It is important to ensure security and privacy of patient's data over the internet. Encryption of patient's data may prove as a viable solution. This paper presents the huge potential in IOT in smart healthcare system

\section{(Yu Lei, Lu Yang and Zhu XiaoJuan,2012)[5]:-}

Presents the application scheme of smart hospital's by using IOT an converting hospitals to smart hospitals. In this paper there are three structures to develop smart hospital. In the first structure it talks about the logical part in which it aims to build an integrated information service platform where all type of application can integrate their services and everyone can easily understand their role through this platform. In second part they have worked on application framework in that there are three layers, composed of perception layer, network layer, data fusion layer. In first layer are added to ensure the integrity of the frame. Network layer is to integrate with each application network in hospitals like cable, internet, communication network etc. Data fusion layer in this layer the integration of identification data is done like patient's name, gender, birth etc. last one is network supporting environment in this network structure adopts the wired and wireless combination structure using technology of IEEE 802.11 a/B/G to access of patient health care by wireless network by indoor AP and as well as outdoor AP.

\section{(S.M.Riazul, Daehan Kwak, MD. Human Kabir, Mahmud Hossain and Kyung-sup kwak,2015)[6]:-}

This paper analyzes distinct IOT security and privacy feature, including security requirements, threat model and attack taxonomies from the health care perspective. In this paper it talks about the all possible vulnerabilities, threats and attack related to IOT domain and how to resolve this problem. To tackle this type of attacks they made a model of intelligent security model for IOT healthcare services and it is designed using dynamic algorithms and collaboration between service and defense against attacks. This model is associated with three security services like protection services are designed to reduce attacks. Detection services activity data from healthcare application, devices, and network and analyzes captured heath data with aid of defense mechanisms, reaction services helps to survive from these attacks.

\section{Proposed work:-}

We here researched that due to less communication between doctor and patient there has been a decline in cautiousness of patients toward some diseases related with kidney and many other problems such as diabetes, high blood pressure and many more so we basically worked on the water retention in kidneys in which we will describe the process how a patient can find his/her water retention in kidney without going to doctor or any pathology lab. This urinalysis test will help patient to find high levels of albumin which can cause hyper tension or chronic kidney disease(CKD).

\section{Data collection:-}

In order to measure water retention kidney we would $1^{\text {st }}$ develop an app on which the patients have to register themselves and after the will be provided with a kit named dip-test. And this data will be collected on cloud server to which only caregivers, doctors and patients have access to it.

\section{After the data collection:-}

After the data has collected and successfully stored on cloud server the doctors or caregivers will get an alert regarding the patients report or urinalysis that they done at their home and then doctors will give there prescription and their views about the present condition of kidney and what precautions and medicines they can take to control it and further this will help in strengthens the bond of patients and doctors and caregivers.

\section{Working of Dip-test:-}

First the user have to download the app in which they have to register themselves and when they get verified they will receive a dip-test kit in which there is a silicon cup, a a colour board which will give the result of their analysis and a dip strip which needs to be dipped in the silicon cup with urine in it and patients have to wait for 2-5 minutes for the colour to change when the colour change the patients can match the colour of the strip to the colour board and they can send it to their doctors using mail or through our app which will store this data and give it to doctor and then doctor can see them and provide necessary precaution to the patients and based on this the patient can get regular check-up at home and get know to the symptoms of the disease. 


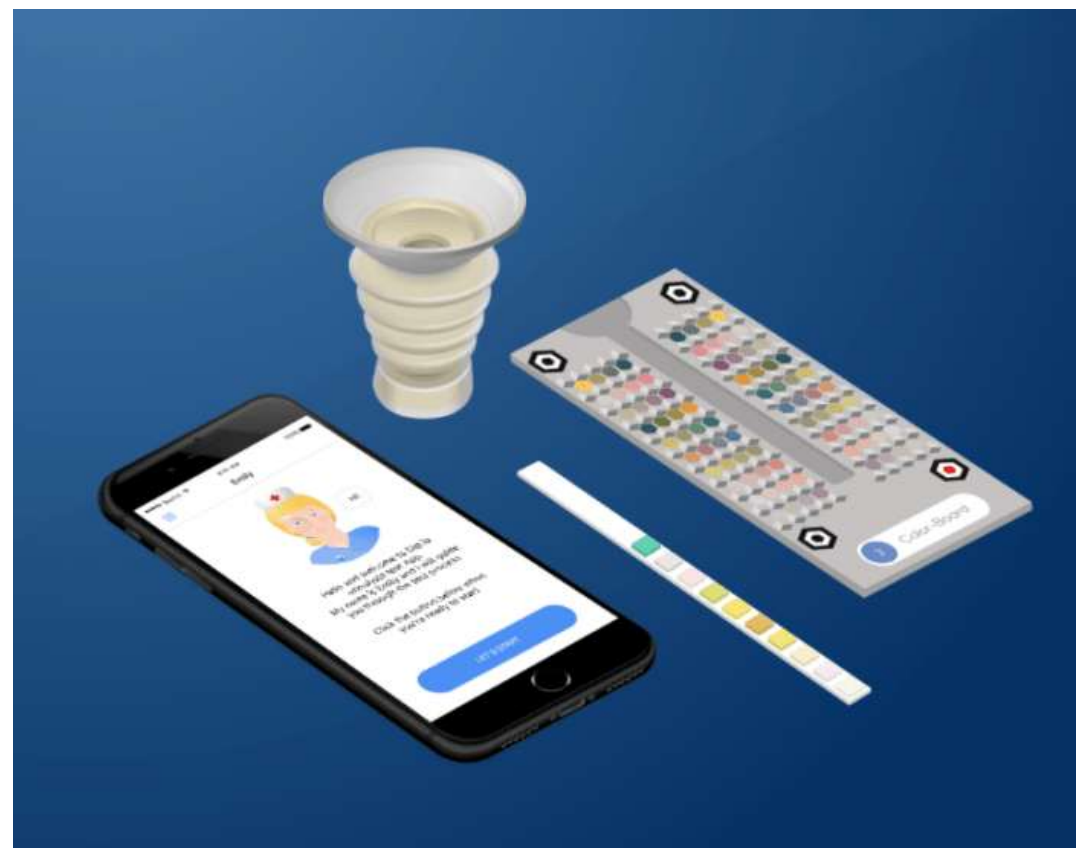

Fig5:-A small representation of our proposed work

\section{Recommendations for Future Works:-}

In our future work we will be placing focus on developing sensors to detect abnormal functioning of kidney. We will develop a model by which patient can check the water retention in his body. This data can be communicated through Bluetooth to a smartphone where after a little processing with dedicated mobile application the data is uploaded to cloud which is made accessible to doctor, patient and caregiver.

Through this model doctor can decide when patient has to appear for dialysis. Which medicine is more efficient? In case of critical situation our model will alert the doctor/caregiver. Lot of research needs to be done to develop sensors which are accurate and with power with the hospital system. Cloud storage is considered a viable solution to for storing large data sets.

Detection of urine can play important role in detection of kidney diseases such as, high creatinine, swelling, CKD and many other that can cause harsh effect on patients health. Several sensors have been made to test the urine but that incompetent to hospital systems.



Fig3:-Model of our future work 


\section{Conclusion:-}

Internet of things is an emerging field in smart health care system. It is important that we Develop quality sensors for wearable technology which are at power with hospital standards. This paper presents technologies currently being used in IOT. A brief information. On patients suffering from kidney failure is discussed with common causes and treatments. This paper proposes a model for detection of kidney failure.

\section{Acknowledgement:-}

We would like to express our sincere gratitude to Accendere Knowledge Management Pvt. Ltd. for providing us the platform and opportunity to pursue the research.

\section{References:-}

1. Stephanie B Baker,Wei iang,Ian Atkinson Internet of thing for Smart Healthcare: Technologies challenges and Opportunities

2. M.Riazul Islam,Daehan Kwak,MD. Humaun Kabir,Mahmud Hossain,Kyung-sup kwak "The internet of things for healthcare: A comprehensive survey.

3. Lei Yu,Yang lu,XiaoJuan Zhu Smart Hospital based on internet of things

4. M.d Miorandi internet of things :Vision,application and challeges

5. Aanchal Khatri, S. Kumar, O. Kaiwartya "Towards Green Computing in Wireless Sensor Networks: Controlled Mobility aided Balanced Tree Approach". International Journal of Communication Systems, 2017.

6. Aanchal Khatri, S. Kumar, Omprakash Kaiwartya, et al. "Optimizing Energy Consumption and Inequality in Wireless Sensor Networks using NSGA-II", In Proceedings of ICCCS, Taylor \& Francis, 10 September, 2016.

7. Mohammad Aazam1, Pham Phuoc Hung2, Eui-Nam Huh3:Cloud of Things: Integrating Internet of Things with Cloud Computing and the Issues Involved

8. Isabel Laranjo, Joaquim Macedo, and Alexandre Santos: Internet of Things for Medication Control: Service Implementation and Testing

9. C. Tokognon, B. Gao, G. Tian and Y. Yan”Structural Health Monitoring Framework Based on Internet of Things"IEEE IoT journal, may 2015.

10. L. Catarinucci, D. Donno, L. Mainetti, L. Palano, L. Patrono, M. Stefanizzi, and L. Tarricone "An IoT-Aware Architecture for Smart Healthcare Systems"IEEE internet of thing journal,2014.

11. C. Hsia, K. Liou, A. Aung, V. Foo, W. Huang, J. Biswas, Analysis and comparison of sleeping posture classification methods using pressure sensitive bed system, in: Annual International Conference of the IEEE Engineering in Medicine and Biology Society, 2009. EMBC 2009, IEEE, 2009, pp. 6131-6134. 